


\title{
El rol de la abducción peirceana en el proceso de la investigación científica
}

\author{
Graciela Velázquez Delgado \\ Universidad de Guanajuato
}

Resumen

En el presente artículo se pretende contribuir al análisis y esclarecimiento de la naturaleza de la abducción peirceana, así como reflexionar sobre su función e importancia en la investigación científica. Para ello, será necesario enunciar primero el pensamiento temprano del filósofo sobre los tres modos de inferencia, para enseguida atender la distinción entre inducción e hipótesis. Por último, nos centraremos en la enunciación y elucidación del razonamiento abductivo para entender el papel protagónico de éste en la investigación científica.

Palabras clave: abducción, Peirce, razonamiento, inferencia, investigación científica.

\section{Abstract}

This article aims to contribute to the analysis and clarification of the nature of the Peircean abduction and think about its role and importance in scientific research. For this, is necessary to first articulate this philosopher early thinking about the three modes of inference, and then concentrate on the distinction between induction and hypothesis. Finally, the analysis will focus on enunciation and elucidation of abductive reasoning has a leading role in scientific research.

Keywords: Abduction, Peirce, Reasoning, Inference, Scientific research. 


\section{Introducción}

C n la actualidad, Charles Sanders Peirce es considerado uno de los intelectos más originales y polifacéticos de los Estados Unidos de América de finales del siglo XIX y principios del XX. Sin embargo, su pensamiento permaneció prácticamente desconocido hasta los años cincuenta del siglo XX, cuando los filósofos de la ciencia se volcaron hacia sus escritos para investigar sobre el razonamiento abductivo. En los últimos años, han proliferado los estudios sobre la obra de este filósofo, lo cual ha propiciado un redescubrimiento de sus ideas y aportaciones. En particular, se ha podido prestar una mayor atención al desarrollo histórico de su pensamiento tardío o de su segunda etapa en la que planteó consistentemente la naturaleza de la abducción. No obstante, hasta hoy día, este planteamiento sigue siendo foco de innumerables controversias tanto en la lógica como en la filosofía de la ciencia. Por todas estas consideraciones, parece muy acertada la opinión de Ian Hacking acerca de Pierce: "deberíamos verlo como un hombre impetuoso, uno de los pocos que entendió los sucesos filosóficos de su siglo y trató de ponerles su sello. No tuvo éxito. No concluyó casi nada, pero comenzó casi todo" (Hacking, 1996: 82).

Peirce distinguió en su sistema lógico tres modos de inferencia: deducción, inducción e hipótesis, poniendo énfasis en la abducción. La reflexión constante sobre los modos de inferencia le hizo considerar que no se trataba de tres operaciones independientes del entendimiento, sino más bien de tres etapas que se entrelazan en el proceso de investigación. La génesis de las hipótesis en el proceso de investigación es el aspecto más novedoso de la teoría peirceana sobre el conocimiento, pues plantea interrogantes específicas sobre el rol de cada uno de los razonamientos en el conocimiento científico. 
Así, en el presente artículo buscamos contribuir al análisis y esclarecimiento de la naturaleza de la abducción peirceana, así como reflexionar su función e importancia en la investigación científica. Para ello, enunciaremos primero su pensamiento temprano sobre los tres modos de inferencia y enseguida realizaremos la distinción entre inducción e hipótesis. Por último, nos centraremos en la enunciación y elucidación del razonamiento abductivo para entender el papel protagónico de éste en la investigación científica.

Para efectos prácticos, este artículo se divide en tres apartados. El primero consiste en un análisis de la tríada inferencial. El segundo aborda la distinción esencial en los razonamientos ampliativos del conocimiento: inducción y abducción. El tercer apartado tiene como tema principal el razonamiento abductivo.

\section{El conocimiento y la tríada inferencial}

Peirce nació en Cambridge (Massachusetts) en 1839. Fue hijo de un matemático llamado Benjamin Peirce, quien le heredó el gusto por la ciencia. Se recibió en química en la Universidad de Harvard en 1859 y pasó la mayor parte de su vida en el instituto United States Coast and Geodetic Survey (1861-1887). Por temporadas cortas, enseñó matemáticas, historia de la filosofía y de las ciencias, así como cursos de lógica en la universidad más prestigiosa de ese estado, la de Harvard. En 1884, fundó su cátedra de Lógica en la Universidad Johns Hopkins de Baltimore, donde se desempeñó desde 1879 hasta que fue separado de su cargo en 1884, a la edad de 45 años, por consejo del comité ejecutivo de dicha institución. Su trabajo lo dio a conocer a través de artículos filosóficos que escribió para revistas prestigiosas de ciencia (como Nation, North American Review, Journal of Speculative Philosophy, Scientific Monthly) y en diccionarios (como Century Dictionary y Dictionary of Philosophy and psychology). Murió en 1914, alejado del ámbito académico en 
Milford (Pennsylvania) sin llegar a publicar -ni siquiera a sistematizar- el grueso de su producción escrita. Después de su muerte, entre 1931 y 1932, comienzan a compilarse sus escritos, publicados posteriormente con el nombre de Collected Papers (Kurtz, 1972: 53).

Con los lógicos de su tiempo, Peirce no tuvo una buena relación, sino un trato sumamente conflictivo. En ese momento, la lógica se había afirmado históricamente como la base de toda ciencia, ya que su materia prima eran los símbolos y su objetivo los principios que conducen a los razonamientos correctos. Peirce consideraba que la Lógica debía dar respuestas válidas a la pregunta: ¿cómo conocemos? Para él, la forma de hacerlo era por medio de la semiótica, la cual estudia la realidad por medio de los signos.

Peirce rechazó el conocimiento intuitivo del racionalismo metafísico cartesiano. Según él, la realidad es externa y más o menos opaca, pero siempre aferrable, y no existe lo completamente incognoscible (Génova, 1997: 29-30). La cuestión del conocimiento intuitivo, en oposición al conocimiento como inferencia, es muy compleja; no obstante, debido al propósito de este artículo, sólo le dedicaremos algunas líneas argumentativas peirceanas. Peirce, en su artículo "Some consequences of Four Incapacities", fundamenta su crítica al cartesianismo en las cuatro negaciones siguientes:

1. No tenemos ningún poder de introspección, sino que todo conocimiento del mundo interno se deriva por razonamiento hipotético de nuestro conocimiento de los hechos externos. 2. No tenemos ningún poder de intuición, sino que toda cognición está determinada lógicamente por cogniciones previas. 3. No tenemos ninguna capacidad de pensar sin signos. 4 . No tenemos ninguna concepción de lo absolutamente incognoscible (CP. 5. 265). ${ }^{1}$

${ }^{1}$ En adelante, todas las citas de Charles Sanders Peirce se referenciarán de esta manera: primero CP, que significa Collected Papers, seguida del volumen y el 
En otro artículo, "Questions Concerning Certain Faculties Claimed for Man" (1868), define lo que entiende por intuición: "A lo largo de este artículo, el término Intuición significará una cognición no determinada por una cognición previa del mismo objeto, y por lo tanto, determinada por algo exterior a la conciencia. [...] Intuición aquí será casi lo mismo que una premisa que no es en sí una conclusión" ( $C P .5$. 213). Génova afirma que la definición anterior está formulada para hacer una distinción entre intuición y conocimiento inferencial, es decir, entre conocimiento inmediato e infalible de la realidad y conocimiento mediado o inferido a partir de conocimientos previos y falibles (Génova, 1997: 33). Para Peirce, todo conocimiento es inferencial, porque a todo conocimiento le precede una cognición. Ahora bien, si atendemos lo que Peirce señala en cuanto a que todo conocimiento es un proceso inferencial desarrollado mediante signos, es decir, mediante argumentos y conocimientos previos, entonces debe haber una serie de cadenas de inferencias o argumentos y, por lo tanto, esta cadena debió tener un comienzo en algún conocimiento no inferencial o intuitivo, o bien, es infinita. La respuesta de Peirce es que debe haber un primum cognitum, una cognición no determinada por una cognición anterior (Génova, 1997: 35). En palabras de Peirce:

Parecería que hay o que hubo; pues poseemos cogniciones, las cuales están determinadas por otras anteriores, y éstas por cogniciones aún más tempranas, entonces debe haber habido una pri-

\footnotetext{
parágrafo de la obra. En el caso de su obra Writings, será $W$, seguida del volumen y el parágrafo. Todas las referencias de la obra de Peirce son traducciones de la autora de este artículo, basadas en los textos originales de las siguientes obras: The Collected Papers of Charles Sanders Peirce (1931), 8 vols., Cambridge, Harvard University Press, editado por Charles Hartshorne y Paul Weiss (versión electrónica); y Writings of Charles S. Peirce: A chronological Edition (1982-2000), 6 vols., Indiana University Press, editado por Peirce Edition Project, Bloomington.
} 
mera en esta serie, o bien nuestro estado de cognición en cualquier momento está completamente determinado, de acuerdo con las leyes lógicas, por nuestro estado en cualquier momento anterior. Pero hay muchos hechos en contra de la última suposición y, por lo tanto, a favor de las cogniciones intuitivas (CP. 5. 259).

Por los postulados anteriores, Cornelius Delaney lo critica y señala que, en primer lugar, Peirce le da más relevancia al aspecto temporal del conocimiento que a su logicidad; en segundo lugar, parece que plantea las cogniciones en una representación lineal y no como una red difusa de interdependencias (Delaney, 1993: 101).

En contraposición a las afirmaciones anteriores de Delaney, si se hace un seguimiento de la teoría peirceana a lo largo de los años se observa que su pensamiento se torna más sistemático a medida que retoma escritos anteriores para generar nuevos. De hecho, su producción no tiene un progreso en el sentido de una evolución lineal, sino un proceso recursivo, donde lo nuevo es el resultado de una constante reelaboración de sus escritos anteriores. Peirce demuestra su teoría con su propia práctica: argumentamos para conocer y todo conocimiento parte de un conocimiento previo que será, a su vez, resultado de otro, provocando un continuum ilimitado.

Después del planteamiento del conocimiento inferencial Peirce se concentró en la clasificación de los modos de inferencia de procedencia aristotélica (de hecho, da por sentado que los tres modos se encuentran en los Analiticos Primeros del estagirita).

Aristóteles, en los "Primeros" y "Segundos analíticos" del Órganon, estableció los principios de la lógica deductiva, pensando principalmente en las ciencias teóricas, en especial en las matemáticas. Consideró en ese momento que por medio de la teoría silogística se podía obtener un método con el cual fuera posible diferenciar entre un razonamiento válido y uno inválido (Stanford Encyclopedia of Philosophy: internet). 
Aristóteles les da una terminología a los tres métodos de razonamiento: apodeixis (deducción), epagogé (inducción) y apagogé (abducción). El método aceptado como válido era el de carácter deductivo (apodeixis) ya que por medio de él era posible llegar a conclusiones necesarias, derivadas efectivamente de sus respectivas premisas. Habló sobre la epagogé o inducción en el capítulo 23 del libro segundo de los "Primeros Analíticos" donde la describe como un proceso contrapuesto a la apodeixis, que es el razonamiento deductivo o apodíctico (Aristóteles, 384/1982). ${ }^{2}$ Para él, la inducción no era un razonamiento válido, pues a partir de las reglas silogísticas la conclusión que se obtiene es falible. Sin embargo, reconoció que los primeros principios de las ciencias se llegaban a establecer por inducción. Es por eso que analiza la inducción partiendo de uno o pocos ejemplos de los que concluye una inducción perfecta. No obstante, esta postulación es muy cuestionable pues significaría que también la inducción sería de carácter necesario; no obstante, para que obtuviera tal estatus, se exigiría la enumeración exhaustiva de los individuos o de los elementos considerados en la formulación silogística. Ahora bien, en el caso de que se llegara a garantizar la enumeración de todos los elementos, el silogismo resultante sería una deducción verdadera, porque las premisas serían universales. La enumeración de todos los elementos por medio de la inducción es imposible de garantizar, como ya lo han demostrado varios filósofos (David Hume y Karl R. Popper, entre otros). En todo caso, lo que puede ofrecer una inducción es una generalización, un salto cualitativo en el conocimiento desde lo particular hasta lo universal. Para Aristóteles, la inducción fue considerada como una guía para la deducción en el proceso de la investigación. En esta idea se encuentra la diferencia de la teoría

${ }^{2}$ En el paréntesis aparece "Aristóteles, 384" para indicar el año o probable año de la edición, seguida de 1982 para indicar el año de la edición consultada para este artículo. 
silogística de Aristóteles y de Peirce, como lo mencionaremos más adelante. También, en el capítulo 25 de los "Primeros Analíticos", hizo referencia a la estructura silogística de la apagogé, razonamiento que consideró inválido por las características que presentaba: la conclusión no era una derivación necesaria de sus premisas, por lo tanto, solamente podían sugerir una conclusión plausible.

Ahora bien, entremos de lleno al análisis de lo que Peirce formuló siguiendo algunos de los postulados de Aristóteles. Para ello, es meritorio mencionar que varios estudiosos de la obra de Peirce, como Karl Otto Apel e Isaac Levi, sostienen que hay dos etapas en las que escribió Peirce: la etapa temprana, de 1861 a 1887, y la etapa madura, de 1888 a 1902 (Apel, 1995: 37). En la etapa temprana, escribió varios artículos que posteriormente modificó en la siguiente. Por tal motivo, estos cambios han dado lugar a que haya una serie de confusiones con respecto a sus planteamientos, como veremos más adelante en el análisis de su concepto de abducción.

Líneas arriba mencionamos que Aristóteles ya había esbozado un tercer tipo de razonamiento: apagogé, que sería posteriormente nombrado por Peirce como hipótesis o abducción. Para el razonamiento hipotético, además del nombre de abducción, usó los de retroducción y presunción, pero fue el de abducción el que predominó, sobre todo en la etapa final de su pensamiento. En su artículo "Kinds of Reasoning", Peirce señala que el término retroducción significa lo mismo que la apagogé aristotélica (CP. 1. 65).

En la etapa temprana de su pensamiento, Peirce escribió varios ensayos donde da cuenta de la tríada inferencial. En estas primeras investigaciones realiza distinciones entre los tipos de argumentos que clasificó como deductivos, inductivos y abductivos. Uno de sus primeros artículos sobre ello fue "On the Natural Classification of Arguments" (CP. 2. 461-516). En este ensayo, ya estaba convencido de que había tres tipos de inferencia. En textos posteriores expone más claramente la tríada inferencial del conocimien- 
to, principalmente en dos artículos: "Deduction, Induction and Hypothesis", de 1878, y “Three Types of Reasoning”, ambos parte de las Cambridge Lectures que dictó en 1903.

En "Deduction, Induction and Hypothesis" sostuvo que el conocimiento se obtiene mediante distintos tipos de inferencias o razonamientos y encontró que el modus Barbara permitía tres diferentes tipos de inferencia: apoidexis (deducción), epagogé (inducción) y apagogé (hipótesis que, posteriormente, en la VI Conferencia de Harvard le daría el nombre de abducción). En este mismo artículo argumenta que la lógica tradicional comúnmente divide los argumentos en dos subclases: las inferencias necesarias o deductivas y las inferencias probables o inductivas (W. 3. 323-325; CP. 2. 619-644).

Para Peirce, siguiendo a Aristóteles, una deducción es la aplicación de una regla o la aplicación de reglas generales a casos particulares. El razonamiento inductivo o sintético es el razonamiento que se obtiene a partir de casos particulares, pero éste no es un silogismo correcto porque no es válido concluir una proposición universal partiendo de dos proposiciones particulares. En el caso de la inducción, aunque la enumeración no sea exhaustiva, de todas formas es posible realizar un salto cualitativo en el conocimiento desde lo particular hasta lo universal, pero sólo aseguraría conclusiones probables. La tercera posibilidad de razonamiento, la inferencia hipotética, es aquélla que de un caso a partir de una regla y un resultado se consigue una conjetura. En este caso, la conclusión obtenida no es segura, sino tan sólo probable, aunque sean ciertas las premisas, porque no es lícito identificar dos sujetos por el hecho de tener un predicado en común (Génova, 1997: 40).

Lo anterior puede ser mejor explicado en el esquema del siguiente cuadro: 


\begin{tabular}{|c|c|c|}
\hline \multicolumn{2}{|c|}{ Inferencias } \\
\hline \multicolumn{1}{|c|}{ Analíticas } & \multicolumn{2}{|c|}{ Sintéticas } \\
\hline Deductivas & Inductivas & Abductivas \\
\hline \multicolumn{1}{|c|}{ Categóricas } & \multicolumn{2}{|c|}{ No categóricas } \\
\hline $\begin{array}{l}\text { Ejemplo: La lógica y las matemá- } \\
\text { ticas contienen reglas que pueden } \\
\text { ser demostradas. El conocimien- } \\
\text { to generado con la demostración } \\
\text { matemática es categóricamente } \\
\text { verdadero. }\end{array}$ & $\begin{array}{l}\text { En las inferencias sintéticas no se } \\
\text { alcanza un conocimiento conclu- } \\
\text { yente o lógicamente válido. }\end{array}$ \\
\hline
\end{tabular}

Cuadro 1. (Del Razo, 2011: 36)

Para Peirce, una deducción no es más que la aplicación de una regla a un caso para establecer un resultado. No obstante, no todas las formas pueden ser reducibles a una deducción. Cada forma de inferencia debe ser expresada de tal manera que se muestren sus características. La deducción es analítica y produce conocimiento categóricamente verdadero, mientras que las inferencias sintéticas, tanto la inducción como la abducción, aportan un conocimiento no categórico, sujeto a constante revisión por su carácter probabilístico. Estos modos de inferencia son ilustrados en su famoso ejemplo de las "alubias":

DEDUCCIÓN

Regla. Todas las alubias en esta bolsa son blancas.

Caso. Estas alubias son de esta bolsa.

Resultado. Estas alubias son blancas.

INDUCCIÓN

Caso. Estas alubias son de esta bolsa.

Resultado. Estas alubias son blancas.

Regla. Todas las alubias de esta bolsa son blancas. 
HIPÓTESIS

Regla. Todas las alubias de esta bolsa son blancas.

Resultado. Estas alubias son blancas.

Caso. Estas alubias son de esta bolsa (W. 3. 325-326; CP. 2. 623).

Después de clasificar esquemáticamente en silogismos las tres inferencias, Peirce se da a la tarea de entrar más a fondo: clarificar la diferencia entre inducción e hipótesis; es decir, reflexionar cuál es la naturaleza de cada una de ellas. Enseguida se tratará esta temática.

\section{Distinción entre inducción e hipótesis}

Ahora bien, a Peirce le interesaba dejar bien clara la diferencia entre inducción e hipótesis porque, como él mismo afirmaba, muchos lógicos de su tiempo seguían considerando que sólo había dos tipos de razonamiento, y creían que no valía la pena explicitar el razonamiento hipotético porque era un razonamiento inválido y daba pie a sostener argumentos sumamente débiles. No obstante lo anterior, Peirce dio mucha importancia a la abducción. En "Pragmatism-the Logic of Abduction" llegó, incluso, a considerar que era la esencia de su pragmatismo, pues las hipótesis son inferencias que nos permiten actuar y formar hábitos para poder conducirnos y tratar de conocer la realidad en sus efectos prácticos (CP. 5. 196).

A diferencia de los lógicos de su tiempo que planteaban que la hipótesis era una inducción, y de varios filósofos que la consideraban una intuición, Peirce la formuló como un tercer razonamiento, no intuitivo, ampliativo del conocimiento e irreductible a la inducción. Esta inferencia tiene una estructura diferente a la deducción e inducción: por un lado, no es una inferencia necesaria como la deducción y, por otro lado, comparte la probabilidad con la inducción. Peirce, en el artículo "Abduction and Perceptual Judgments", dice en cuanto a lo formal de la abducción que "aunque 
está muy poco obstaculizada por reglas lógicas, no obstante es una inferencia lógica que afirma su conclusión sólo problemáticamente o conjeturalmente, es verdad, sin embargo, tiene una forma lógica perfectamente definida” (CP. 5. 188). En la abducción se busca pasar del efecto a la causa, es decir, de la observación de ciertos casos a la suposición de un principio general que pueda explicarlos (Beuchot, 1998: 58).

En "Deduction, Induction and Hypothesis", Peirce clasificó la inferencia deductiva como analítica y la inducción e hipótesis como sintéticas. La inferencia analítica es llamada también explicativa, pues no hace sino explicitar lo que ya está en las premisas, y la sintética es ampliativa, porque ańade nuevos conocimientos. En este caso, la inducción y la hipótesis están dentro de una misma subclase: ampliativa. Así, debe haber semejanzas, pero también diferencias: "la inducción se da cuando generalizamos a partir de un número de casos de lo que algo es verdad, e inferimos que la misma cosa es verdad de una clase entera. O bien, cuando encontramos que cierta cosa es verdadera de cierta proporción de casos e inferimos que es verdad la misma proporción de la clase entera" (CP. 2. 624). En el caso de la hipótesis, dice que "se da cuando encontramos alguna circunstancia muy curiosa, que se explicaría por la suposición de que fuera un caso de cierta regla general, y en consecuencia adoptamos esa suposición. O bien, cuando constatamos que en ciertos aspectos dos objetos tienen un fuerte parecido, e inferimos que se asemejan entre sí marcadamente en otros aspectos" (CP. 2. 624).

En este sentido, las semejanzas entre dos o más sujetos nos hacen conjeturar que dichas similitudes van más allá de lo meramente observado y, a partir de ello, se logra un razonamiento hipotético. En cambio, la inducción se establece con las observaciones de un carácter que comparten algunos individuos de una clase $y$, con ello, generalizamos ese carácter al resto de los individuos no 
observados de la clase (Génova, 1997: 48). Por lo tanto, la inducción y la hipótesis son ampliativas, en cuanto ambas extienden el conocimiento más allá de lo meramente observado. Peirce, en su búsqueda de clarificación para las dos inferencias, señala:

Por medio de la inducción, concluimos que hechos similares a los hechos observados son verdaderos en casos no examinados. Mediante la hipótesis, concluimos la existencia de un hecho muy diferente de cualquier cosa observada, de la cual, según las leyes conocidas, resultaría necesariamente algo observado. El primero es un razonamiento de los particulares a la ley general; el segundo, del efecto a la causa. El primero clasifica, el otro explica (CP. 2. 636).

Ante tales conclusiones, admite que hay cierto paralelismo entre las dos formas de razonamiento, pero la hipótesis es irreductible a la inducción, por lo tanto, deben ser de naturaleza diferente. Con el ejemplo siguiente, Peirce muestra puntualmente tal distinción:

Cierto anónimo escrito en un trozo de papel desgarrado. Se sospecha que el autor es cierta persona. Su escritorio, al que solamente él ha tenido acceso, es registrado y en él se encuentra un trozo de papel, cuyo borde desgarrado se ajusta exactamente en todas sus irregularidades con el del papel en cuestión. Es una inferencia hipotética admisible que el hombre sospechoso fuera efectivamente el autor. La base de esta inferencia, evidentemente, es que es poco probable que dos piezas de papel puedan encajar perfectamente por accidente [...] La inferencia desde el contorno del papel hasta su propietario es precisamente lo que distingue la hipótesis de la inducción y la convierte en un paso más audaz y arriesgado (CP. 2. 632).

En el ejemplo anterior de inferencia hipotética se advierte también una inferencia inductiva enumerativa, misma que Peirce describe como "un cierto número de caracteres pertenecientes a cierta clase 
que se encuentran en el objeto, de donde se infiere que todos los caracteres de esa clase pertenecen al objeto en cuestión" (CP. 2. 632). El argumento de la inferencia inductiva enumerativa sería que de la observación de un número de hechos en torno al desgarramiento de las hojas de papel se puede inferir, por tanto, que los contornos de los dos o más trozos de papel deben coincidir. El fundamento de esta inferencia hipotética radica, evidentemente, en que es inverosímil que dos trozos rotos de papel coincidan por accidente. Por su parte, la inferencia abductiva se concentra en el salto lógico que va desde el contorno del papel hasta su propietario. Este salto lógico es, precisamente, lo que distingue la hipótesis de la inducción.

A pesar de resaltar la distinción, Peirce reconoce que su paralelismo sigue confundiéndolas. Una de las razones es que tanto la hipótesis como la inducción amplían el conocimiento de lo observado a lo inobservado, aunque en forma diferente. Otra razón es que las dos comparten, en cierto grado, la misma naturaleza, pues lo que es conocido por medio de la observación en unos individuos o cosas se supone y se generaliza en otros. Al respecto, Peirce menciona:

La gran diferencia entre la inducción y la hipótesis es que la primera infiere la existencia de fenómenos tales como los que hemos observado en casos similares, mientras que la hipótesis supone algo de tipo distinto a lo que hemos observado directamente, y frecuentemente algo que nos sería imposible observar directamente. En consecuencia, cuando extendemos una inducción mucho más allá de los límites de nuestra observación, la inferencia participa de la naturaleza de la hipótesis. Sería absurdo decir que tenemos una garantía no inductiva para una generalización extendida un poco más allá de los límites de la experiencia y no hay una línea más allá de la cual podamos empujar nuestras inferencias; se vuelve más débil cuanto más se empuja. Si una inducción se lleva demasiado lejos, no podemos darle mucho crédito, a no ser 
que comprobemos que tal ampliación explica algún hecho que podemos y hemos observado. Aquí, pues, tenemos una especie de mezcla de inducción e hipótesis, apoyándose recíprocamente (CP. 2. 640).

Como puede observarse hasta este momento, Peirce no había llegado a separar tajantemente las dos inferencias, debido a que cada una participa de la naturaleza de la otra. Más tarde ampliará su noción de inducción, mientras que la hipótesis se convierte en abducción. Esto se advierte con toda claridad cuando formula la lógica de la investigación que se analiza enseguida.

\section{La abducción en el proceso de la investigación científica}

Después de que Peirce se retiró de la vida académica y científica en la década 1890-1900, la teoría peirceana sobre la abducción sufrió un cambio gradual (Fann, 1970: 28). El cambio fue así: en primer lugar, las tres formas de inferencia se integran para formar las tres etapas de la investigación en el método científico; en segundo lugar, realiza una distinción más sistemática entre abducción e inducción, perfilándose ya la abducción como la única inferencia ampliativa y creativa. A partir de este momento, las diferentes formas de denominación como hipótesis e inferencia hipotética se sustituyen por la de abducción, así como retroducción, para definir de mejor manera su naturaleza de "razonamiento hacia atrás". En Minute Logic (1902) escribe lo siguiente:

Sobre este tema, mi doctrina ha sido inmensamente mejorada desde mi ensayo 'una teoría de la inferencia probable' publicado en 1883. En lo que yo decía allí acerca de la 'inferencia hipotética' era un explorador en un terreno inexplorado [...]. Mi error capital fue negativo, no percibir que, de acuerdo con mis propios principios, el razonamiento que estaba examinando no podía 
ser el razonamiento por medio del cual adoptamos una hipótesis [...]. Pero yo estaba demasiado aferrado a la consideración de las formas silogísticas y a la doctrina de la extensión y comprensión lógicas, a las que hice más fundamentales de lo que realmente son. Mientras sostuve esa opinión, mis concepciones de la abducción necesariamente confundieron dos tipos de razonamiento diferentes (CP. 2. 102).

En lo anterior, Peirce alude a los dos tipos de razonamiento. La hipótesis que menciona se convierte en abducción o retroducción, mientras que la inducción de caracteres o inducción cualitativa se clasificará en adelante como diferentes tipos de inducción. Ya en su etapa madura, en la lectura V de 1903 "The Three kinds of Goodness", consideró que la única inferencia ampliativa era la abducción, pues la inducción "nunca puede originar ninguna idea. Tampoco la deducción. Todas las ideas de la ciencia llegan en forma de abducción" (CP. 5. 145). En esta etapa, plantea que las tres inferencias no son sólo tipos de razonamientos sino tres etapas que colaboran paralelamente de forma consistente y sistemática en el proceso de la investigación científica. Esta idea la expone en 1903, en Lecturas sobre el Pragmatismo, dictadas en Harvard, específicamente en la Lectura VI "Three Types of Reasoning":

La abducción es el proceso de formación de una hipótesis explicativa. Es una operación lógica que introduce una nueva idea; la inducción no hace más que determinar un valor y la deducción involucra solamente consecuencias necesarias de una hipótesis pura. La deducción provee algo que debe ser; la inducción muestra que algo es realmente operativo; la abducción simplemente sugiere que algo puede ser.

Es solo la justificación de que la deducción puede sugerir una predicción que puede ser probada por la inducción, y que si queremos aprender algo o entender el fenómeno, debe ser por abducción por la que se produjo (CP. 5. 171; Minnamier, 2004: 78). 
A partir de este momento, Peirce postula la importancia de la abducción en el procedimiento metodológico de la investigación que comprende las tres etapas caracterizadas por las tres inferencias (CP. 2. 775; CP. 5. 170, CP. 6. 100). A la deducción le corresponde el mayor grado de certeza, porque es una inferencia necesaria pero el menor grado de fertilidad explicativa. La inducción confirma experimentalmente la hipótesis en una determinada proporción de casos y a la abducción le corresponde llevar nuevas ideas e introducir la creatividad en la ciencia. Puede pensarse gráficamente como un proceso recursivo donde el rol de la abducción es traer nuevas ideas para formular una hipótesis; luego el papel de la deducción es derivar las consecuencias de lo asumido en una cierta teoría, por lo tanto, estas consecuencias son lógicamente necesarias y pueden ser deducidas de la validez de la premisas; por último, la tarea de la inducción consiste en comenzar una teoría, deduciendo las predicciones de un fenómeno y observándolos en orden para ver si concuerdan con la teoría (Minnamier, 2004: 79). Además de afirmar que la abducción tiene una forma lógica, Peirce defendió que era parte de un procedimiento auto-correctivo de indagación y construcción de conocimiento (CP. 7. 59). La forma de la inferencia es ésta:

-El sorprendente hecho $\mathrm{C}$, es observado.

-Pero si A fuera verdadera, C podría ser una cosa corriente (matter of course ${ }^{3}$ ).

-Por lo tanto, hay razones para sospechar que A es verdadera (CP. 5. 189).

Por medio de hipótesis o conjeturas, la abducción proporciona la pista que el científico debe seguir para realizar la experimentación

${ }^{3}$ Este concepto se mantiene en inglés por dificultades de traslado al español. 
y, al mismo tiempo, le indica cuál es el experimento que debe verificar por medio de la inducción. Así, pues, la inferencia abductiva propone una hipótesis que debe ser en sí misma verosímil y aceptable, pero no da ninguna otra garantía. No es más que una sugerencia plausible de la cual se derivan consecuencias en una teoría y la comprobación sólo puede darse por medio de la inducción. En este sentido, la abducción, aun siendo el origen de la explicación, es un argumento incompleto, puesto que no puede por sí misma establecer conclusiones plausibles sin el complemento de la deducción y la inducción. Peirce sostiene:

La abducción, por otra parte, es meramente preparatoria. Es el primer paso del razonamiento científico, así como la inducción es el paso concluyente [...] Abducción e inducción tienen ciertamente esta característica común, que ambas conducen a la aceptación de una hipótesis porque los hechos observados son tales como resultarían si fueran consecuencias necesarias o probables de la hipótesis. No obstante, por lo demás, son los polos opuestos de la razón, uno el más ineficaz, el otro el más efectivo de los argumentos (CP. 7. 218).

Para Peirce, la investigación comienza cuando el científico se encuentra con un hecho sorprendente y para explicarlo construye instintivamente una o varias hipótesis, para luego seleccionar una como la más plausible. Para Peirce, no hay una clara distinción entre la construcción y la selección de hipótesis en el proceso de investigación antes de pasar a las etapas siguientes (la deducción de consecuencias experimentables y su confirmación inductiva) (CP. 8. 209). Así lo argumenta:

Ahora procederé a considerar los principios que deben guiarnos en la abducción, o el proceso de elegir una hipótesis. [...] Ahora, la cuestión es que una nueva verdad no puede venir de la inducción 
o de la deducción. Sólo puede llegar de la abducción; la abducción, después de todo, no es otra cosa que conjeturar. Estamos pues obligados a esperar que, aunque las posibles explicaciones de los hechos pueden ser estrictamente innumerables, aún así nuestra mente será capaz, en algún número finito de conjeturas, de conjeturar una sola explicación verdadera de ellos. Estamos obligados a asumir, independientemente de cualquier evidencia de que es verdadera. Animados por esta esperanza, debemos proceder a la construcción de una hipótesis (CP. 7. 219; las cursivas son mías).

De acuerdo con Peirce, en la actividad científica hay una hipótesis fundamental y primaria con la que se inicia el proceso de investigación. Por supuesto, una hipótesis es sometida a un proceso de evaluación y experimentación, siempre y cuando, previamente, exhiba que es plausible; es decir, que dé cuenta de inicio que puede llevar a buen puerto una solución para los fenómenos. En este proceso de investigación, la abducción sugerirá una hipótesis y se desplegarán sus consecuencias deductivas para probarla mediante un testeo inductivo (CP. 2. 511). Por hipótesis plausible, Peirce entiende "a aquella teoría que todavía no ha sido sujeta a ninguna prueba, por lo tanto, más o menos puede explicar fenómenos sorprendentes si fuera verdadera y que se recomienda a sí misma para un examen posterior" (CP. 2. 662).

La distinción entre la construcción y selección de hipótesis funciona para los que hacen una división tajante entre el contexto de descubrimiento y el contexto de justificación, pero no para la lógica de Peirce. En la postura tradicional de la lógica sólo habría postulados lógicos en la selección de hipótesis, mientras que la construcción sería algo inexplicable; sin embargo, Peirce considera que un hecho sorprendente puede ser explicado por medio de la hipótesis que el científico construye. Así, tanto la construcción como la selección de la mejor hipótesis tienen el mismo propósito: explicar ciertos hechos. En conclusión, se observa que para Peirce 
la construcción y selección están íntimamente conectadas, se funden en una sola a lo largo del proceso de investigación.

Ahora bien, es importante señalar que Peirce subraya el carácter tentativo y provisorio de la hipótesis adoptada a partir de la aplicación de varios principios de plausibilidad. Para él, la "retroducción es la adopción provisional de una hipótesis porque cada consecuencia posible de ella es capaz de una verificación experimental" (CP. 1. 68). Sin embargo, el hecho de que una hipótesis explique los fenómenos para los que fue propuesta, no es una condición suficiente para su aceptación. Es más, la condición que autoriza a una hipótesis es que luego ésta "sea comprobada por comparación con la observación” (CP. 1. 121). Como él mismo menciona: "la retroducción no establece seguridad; la hipótesis debe ser probada” (CP. 6. 470). Por lo tanto, en este proceso la abducción participa tanto en la construcción como en la evaluación de las hipótesis. Con todos los criterios formales de la abducción, Peirce trata de "reconciliar lo universal con lo particular, y proporciona una sistematización del conocimiento que lleve a superar tanto el relativismo caótico como el universalismo ingenuo, admitiendo la vaguedad, y tratando de reducirla lo más posible después de haberla reconocido en la realidad" (Beuchot, 1998: 63).

\section{Conclusiones}

A lo largo de este artículo se pudo observar que Peirce indagó de manera profunda sobre los razonamientos y fases de la investigación científica. A diferencia de los lógicos de su tiempo, Peirce consideró que había tres tipos de inferencias en las que se sustenta no sólo el razonamiento, sino el proceso de la investigación cientí- 
fica. Por supuesto, la lógica tradicional no dio cabida a este tipo de planteamientos, pues esto hubiera significado tambalear las estructuras lógicas que sustentaban el método científico.

Por tanto, en la lógica tradicional nunca se reconoció la importancia de la abducción en el proceso de investigación científica. Así, este razonamiento quedó eclipsado ante la hegemonía de los dos razonamientos reconocidos por los científicos como los únicos que garantizan la validez de sus investigaciones. Por fortuna, después de la primera mitad del siglo XX muchos lógicos, filósofos y científicos han dado preeminencia al razonamiento abductivo, por lo que ello se ha vuelto a revisar la obra de Peirce donde postula que el proceso de la investigación científica comienza con la abducción, seguida de las consecuencias deductivas de la teoría y del testeo experimental de la inducción.

Por supuesto, al reconocer la importancia de la abducción como el razonamiento que inicia el proceso de la investigación científica y la única que permite la creatividad en ella, se le da un estatus diferente en la lógica. Ya no se le toma de manera negativa como una inferencia sumamente débil, sino que es considerada con una fertilidad explicativa que inicia y propone el camino a seguir en la investigación científica. Claro que si se reconoce lo anterior, también deben considerarse consecuencias teóricas para la investigación filosófica y científica en la que hay una re-definición de la noción de verdad, que en el modelo peirceano será entendida no como una mera propiedad de la proposición, que consiste en la correspondencia con los hechos o en la coherencia con otras proposiciones, sino como un consenso alcanzado en colectividad de los científicos. Este consenso es lo que denominó Peirce como su máxima pragmática, la cual postula que el modo de hacer claras nuestras ideas acerca de la realidad descansa sobre dos pilares: la experiencia y la comunidad. Ello permite formular no sólo las condiciones formales para la indagación científica, sino, además, 
una dimensión social de la realidad orientada por los hábitos interpretativos.

\section{Bibliografía}

Aristóteles, 1982, Tratados de Lógica. El Organom, México, Porrúa. Apel, Karl Otto,1995, Charles S. Peirce: From pragmatism to pragmaticism, John Michael Krois (trad.), New Jersey, Humanities Press.

Beuchot, Mauricio, 1998, "Abducción y analogía”, Analogía Filosófica, vol. 12, núm. 1, pp. 57-69, disponible en: http://www. unav.es/gep/AN/ANIndice.html (consultado el 15/VIII/2013).

Delaney, Cornelius, 1993, Science, Knowledge and Mind. A Study in the Philosophy of C.S. Peirce. Notre Dame, University of Notre Dame Press.

Del Razo Canuto, Carlo, 2011, Razonamientos evidenciales en arqueología: abducción e inferencia a la mejor explicación (tesis para obtener el grado de Maestro en Humanidades: Area Filosofía de la ciencia), México, UAM-I.

Fann, K.T., 1970, Peirce's Theory of Abduction, La Haya, Martinus Nijhoff.

Génova Fuster, Gonzalo, 1997, Charles Peirce: la lógica del descubrimiento, Cuaderno de Anuario filosófico 45, Universidad de Navarra, disponible en: file://C:/Users/Vaio/Downloads/Genova.pdf (consultado el 5/X/2013).

Hacking, Ian, 1996, Representar e intervenir, México, Paidós/ UNAM.

Kurtz, Paul, 1972, Filosofía norteamericana en el siglo XX. México, FCE. 
Minnamier, Gerhard, 2004, "Peirce-Suit of Truth: Why Inference to the Best Explanation and abduction ought not to be confused", Erkenntnis, vol. 60, núm. 1, pp. 75-105.

Murphey, Murray, 1993, The Development of Peirce's Philosophy, Indianapolis, Hackett.

Peirce, Charles Sanders, 1931, The Collected Papers of Charles Sanders Peirce, Vol. 1, Principles of Philosophy, [versión electrónica], Charles Hartshorne y Paul Weiss (eds.), Cambridge MA, Harvard University Press.

, 1932, The Collected Papers of Charles Sanders Peirce, Vol. 2, Elements of Logic, [versión electrónica], Charles Hartshorne y Paul Weiss (eds.), Cambridge MA, Harvard University Press.

, 1933, The Collected Papers of Charles Sanders Peirce, Vol. 3, Exact Logic (Published Papers), [versión electrónica], Charles Hartshorne y Paul Weiss (eds.), Cambridge MA, Harvard University Press.

, 1933, The Collected Papers of Charles Sanders Peirce, Vol. 4, The Simplest Mathematics, [versión electrónica], Charles Hartshorne y Paul Weiss (eds.), Cambridge MA, Harvard University Press.

, 1934, The Collected Papers of Charles Sanders Peirce, Vol. 5, Pragmatism and Pragmaticism, [versión electrónica], Charles Hartshorne y Paul Weiss (eds.), Cambridge MA, Harvard University Press.

, 1935, The Collected Papers of Charles Sanders Peirce, Vol. 6, Scientific Metaphysics, [versión electrónica], Charles Hartshorne y Paul Weiss (eds.), Cambridge MA, Harvard University Press. , 1958a, The Collected Papers of Charles Sanders Peirce, Vol. 7, Science and Philosophy, [versión electrónica], Arthur W. Burks (ed.), Cambridge MA, Harvard University Press. 
1958b, The Collected Papers of Charles Sanders Peirce, Vol. 8, Reviews, Correspondence, and Bibliography, [versión electrónica], Arthur W. Burks (ed.), Cambridge MA, Harvard University Press.

,Writings of Charles S. Peirce: A chronological Edition, Vol. 1, 1857-1866, Max H. Fisch (General Editor), Bloomington, Peirce Edition Project. Indiana University-Purdue University Indianapolis.

,Writings of Charles S. Peirce: A chronological Edition, Vol. 2, 1867-1871, Edward C. Moore (Editor), Bloomington, Peirce Edition Project. Indiana University-Purdue University Indianapolis.

,Writings of Charles S. Peirce: A chronological Edition, Vol. 3, 1872-1878, Max H. Fisch (Senior Editor), Bloomington, Peirce Edition Project. Indiana University-Purdue University Indianapolis.

,Writings of Charles S. Peirce: A chronological Edition, Vol. 4, 1879-1884, Max H. Fisch (Senior Editor), Bloomington, Peirce Edition Project. Indiana University-Purdue University Indianapolis.

Writings of Charles S. Peirce: A chronological Edition, Vol. 5, 1884-1886, Max H. Fisch (Senior Editor), Bloomington, Peirce Edition Project. Indiana University-Purdue University Indianapolis.

,Writings of Charles S. Peirce: A chronological Edition, Vol. 6, 1886-1890, Nathan Houser (General Editor), Bloomington, Peirce Edition Project. Indiana University-Purdue University Indianapolis.

Standford Encyclopedia of Philosophy, disponible en: http://plato. stanford.edu/ (consultada el 7/IX/2013).

Recibido: 10 de enero de 2014 Aceptado: 16 de noviembre de 2014 
El rol de la abducción peirceana en el proceso de la investigación científica 